\title{
Caractéristiques sociodémographiques des éleveurs de porcs (Sus Scrofa domesticus) et structure du cheptel porcin au Sud du Bénin
}

\author{
D. DJIMENOU ${ }^{1,2^{*}}$, H. ADOUKONOU-SAGBADJA ${ }^{2}$, D. O. KOUDANDE ${ }^{1}$, \\ C. A. A. M. CHRYSOSTOME ${ }^{3}$, S. M. HOUNZANGBE-ADOTE ${ }^{4}$ et C. AGBANGLA ${ }^{5}$ \\ ${ }^{1}$ Laboratoire de Recherches Zootechniques, Vétérinaires et Halieutiques (LRZVH), \\ Institut National des Recherches Agricoles du Bénin, 01 BP 884 Cotonou, Bénin. \\ ${ }^{2}$ Laboratoire des Ressources Génétiques et Amélioration des Espèces (REGAME), \\ Faculté des Sciences et Techniques de l'Université d'Abomey-Calavi, BP 1947 Abomey-Calavi, Bénin. \\ ${ }^{3}$ Laboratoire de Recherche Avicole et de Zoo-Economie (LARAZE), \\ Faculté des Sciences Agronomiques de l'Université d'Abomey-Calavi, 01 BP 2770 Cotonou, Bénin. \\ ${ }^{4}$ Laboratoire d'Ethnopharmacologie et de Santé Animale (LESA), Faculté des Sciences Agronomiques de \\ l'Université d'Abomey-Calavi, 01 BP 526 Cotonou, Bénin. \\ ${ }^{5}$ Laboratoire de Génétique Moléculaire et Analyse des Génomes (LGMAG), \\ Faculté des Sciences et Techniques de l'Université d'Abomey-Calavi, BP 142 Abomey-Calavi, Bénin. \\ *Auteur correspondant ; E-mail : ndavidjimenou@gmail.com, (+229)95696381/62402221.
}

\section{RESUME}

L'élevage porcin en milieu paysan, une activité économiquement rentable, est peu explorée au Bénin. En vue de déterminer les caractéristiques des producteurs et la structure des cheptels porcins au Sud du Bénin, une enquête transversale et rétrospective couplée aux observations directes des cheptels a été conduite auprès de 105 éleveurs de porcs. Les résultats ont montré que les éleveurs appartenaient à trois grands groupes sociolinguistiques dont les Fon, les Adja et les Yoruba, et étaient dominés par les artisans et les agriculteurs. Ils étaient en majorité des hommes et chefs de ménage. Leur âge a varié entre 33 et 58 ans et leur expérience entre 10 et 20 ans. La motivation des éleveurs était liée à la rusticité et la productivité des porcs. La race de porc majoritairement élevée était le porc local. La taille du troupeau variait entre 10 et 23 têtes. Les cheptels regorgeaient des porcs de tous les stades physiologiques à part les verrats qui étaient en nombre réduit. L'élevage porcin est l'apanage de presque tous les groupes sociolinguistiques et socioprofessionnels au Sud du Bénin. Un appui politique au profit de cette activité sera un grand atout pour la réduction de la pauvreté au Bénin.

(C) 2017 International Formulae Group. All rights reserved.

Mots clés : Caractéristiques des éleveurs, élevage de porcs, enquête transversale et rétrospective, porc local, Sud-Bénin. 


\title{
Socio-demographic characteristics of pig producers and structure of pig herd in South of Benin
}

\begin{abstract}
Pigs breeding in rural areas, an economically profitable activity, is less explored in Benin. To determine the characteristics of producers and the structure of pig herds in South of Benin, a transverse and retrospective investigation coupled with direct observations of pig herds was led using 105 pig breeders. Results showed that breeders belonged to three great sociolinguistic groups comprising Fon, Adja and Yoruba, and were dominated by craftsmen and farmers. Breeders were in majority men and heads of household. Their age varied between 33 and 58 years and their practice between 10 and 20 years. Breeders' motivation was related to the rusticity and the productivity of pigs. The local pig was the mostly used breed. The herds size varied between 10 and 23 heads. Herds were composed of pigs at different physiological stages except boars that were of reduced number. Pigs breeding is a prerogative activity of almost all sociolinguistic and socio-professional groups in South of Benin. Therefore, a political support of this activity will be a large asset for the reduction of poverty in Benin.
\end{abstract}

(C) 2017 International Formulae Group. All rights reserved.

Keywords: Breeders' characteristics, local pig, pigs breeding, South of Benin, transverse and retrospective survey.

\section{INTRODUCTION}

Le contexte économique globalement difficile pour les petits producteurs des zones rurales et urbaines d'Afrique subsaharienne (ASS) oblige à une diversification des activités pour pallier les difficultés économiques (Mopaté et Kaboré-Zoungrana, 2010). Ainsi, l'élevage de porcs, par ses multiples avantages (cycle de reproduction court et forte prolificité) par rapport aux autres animaux d'élevage, est tout indiqué pour réduire la pauvreté et la malnutrition. La production de viande porcine en ASS, stimulée par la forte urbanisation est de 800 000 tonnes (Porphyre, 2009). Cet accroissement de la production est surtout l'œuvre des petites unités familiales qui produisent $90 \%$ de la viande porcine, basée sur l'utilisation des sous-produits domestiques et agro-industriels (Boutonnet et al., 2000). L'élevage de porcs au Bénin est largement pratiqué par les populations en milieu paysan comme en milieu urbain et périurbain. Ces éleveurs de porcs étaient organisés en
Association Nationale des Eleveurs de Porcs (ANEP) créé au lendemain du ravage de l'épidémie de Peste Porcine Africaine (PPA) au Bénin en 1997. Cette association a eu des problèmes de fonctionnement, et malgré les difficultés qu'ils rencontrent dans le soussecteur, les éleveurs se sont efforcés à répondre aux besoins des consommateurs de viande porcine. La concentration de la population dans les centres urbains entraîne une forte demande en produits animaux (Mopaté et Kossou, 2002). La prolificité élevée du porc (Youssao et al., 2009) le classe parmi les espèces de choix pour répondre rapidement à cette demande, mieux que d'autres animaux à cycle court comme les petits ruminants. La consommation de la viande de porc connaît un regain au Bénin, particulièrement dans les régions du Sud du pays où les interdits religieux ont très peu d'influence (Atodjinou et Dotcho, 2006). La multiplication des maquis et restaurants de la viande de porcs et l'affluence qu'ils 
connaissent (Aboki, 2011) témoignent de la forte demande en viande de porcs au Bénin.

Dans le but de satisfaire ce besoin sans cesse croissant, il urge de mieux organiser la filière porcine afin d'assurer la disponibilité permanente des matières premières dont ont besoin ces maquis et restaurants. $\mathrm{La}$ connaissance des acteurs impliqués dans la production et la commercialisation des porcs vivants est un des préalables de l'organisation cette filière. C'est dans ce contexte que la présente étude est initiée. Elle vise spécifiquement à déterminer les caractéristiques sociodémographiques des éleveurs de porcs et la structure du cheptel porcin au Sud du Bénin.

\section{MATERIEL ET METHODES}

\section{Enquête et collecte des données}

Une enquête transversale et rétrospective couplée avec des observations directes sur les élevages et effectifs porcins a été utilisée. Des rendez-vous ont précédé les entretiens avec les éleveurs sous forme de prétest du matériel de collecte des données. La phase de collecte des données s'était déroulée dans sept départements au Sud du Bénin (Atlantique, Littoral, Mono, Couffo, Ouémé et Plateau) et plus précisément dans la commune la plus productrice de porc au niveau de chaque département selon les statistiques de CountryStats (2013), (Figure 1). Au total, 105 éleveurs dont 15 par département ont été identifiés au hasard pour la collecte des données à l'aide d'un questionnaire structuré. Les entretiens et observations ont eu lieu soit tôt le matin soit le soir en fin de journée, qui sont les moments propices pour accéder aux éleveurs sans perturber leurs activités quotidiennes. Les points abordés pour la collecte des données sont relatifs aux lieux d'implantation des élevages, aux caractéristiques des producteurs, au mode de constitution du troupeau, aux races de porc élevées, aux autres espèces animales associées, à la production agricole associée et la surface emblavée, à la taille et la structure du troupeau. Des marqueurs ont été utilisés pour éviter toute erreur lors du comptage des animaux.

\section{Analyses statistiques}

Les données collectées ont été dépouillées et transférées dans une base de données conçue dans le logiciel de traitement statistique Sphinx V 4.5. La statistique descriptive a été utilisée pour le calcul des fréquences. Le test de Chi-2 d'indépendance a été effectué pour l'analyse des variables qualitatives (sexe, activités principale et secondaire, situation matrimoniale, niveau d'instruction, niveau de prospérité, groupe socioculturel, motivation pour le porc, statut $\mathrm{du}$ propriétaire, mode de constitution du troupeau, race de porc élevée, autres espèces animales associées, production agricole associée). Pour les variables quantitatives (âge, expérience dans l'élevage de porcs, superficie emblavée, effectifs des animaux par classe d'âge, taille des troupeaux), une analyse de variance à un seul facteur a permis d'apprécier la significativité de l'effet département au seuil de 5\%. Les comparaisons entre les moyennes ont été faites deux à deux par le test de t. Ensuite, des analyses croisées entre groupes socioprofessionnels et taille du troupeau, groupes socioprofessionnels et race de porcs élevée, sexe de l'éleveur et taille du troupeau, sexe de l'éleveur et race de porcs élevée ont été faites pour apprécier l'influence des caractéristiques des éleveurs sur l'effectif des cheptels et le choix de la race de porc élevée. 


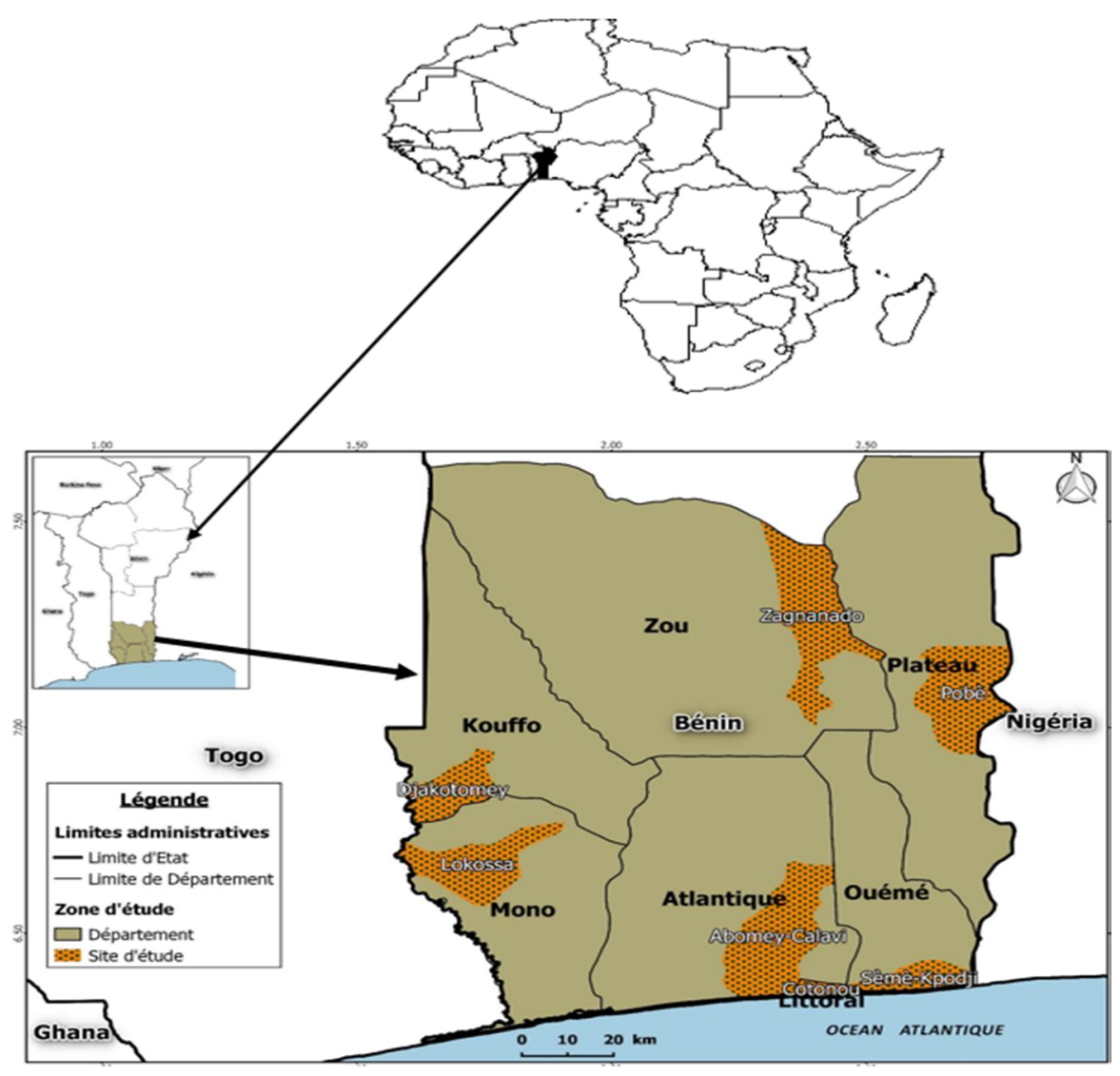

Figure 1: Situation géographique des milieux d'étude (Données du terrain (2015) et base de données DIVA-GIS).

\section{RESULTATS}

Groupes sociolinguistiques des éleveurs de porcs au Sud du Bénin

L'élevage de porcs est pratiqué par trois grands groupes sociolinguistiques au Sud-Bénin tels que les Fon les Adja et les Yoruba (Figure 2) qui ont varié d'un département à un autre avec une différence très hautement significative $(p<0,001)$. Dans les départements de l'Atlantique, le Littoral, l'Ouémé et dans le Zou, tous les éleveurs de porcs appartenaient au groupe sociolinguistique Fon. Les éleveurs du groupe sociolinguistique Adja étaient dominants dans leurs zones de distribution d'origine (le Mono et le Couffo). Seulement $16 \%$ des éleveurs du Mono étaient Fon. Ceux du département du Plateau étaient dominés par les Yoruba et comprenaient dans une moindre mesure des Fon.

\section{Sexe et groupes socioprofessionnels des éleveurs de porcs au Sud du Bénin}

Le Tableau 1 présente le sexe et les groupes socioprofessionnels des éleveurs. Il ressort de ce Tableau que les éleveurs de porcs du Sud-Bénin étaient en majorité des hommes $(79 \%)$ par rapport aux femmes $(21 \%)$, exceptée dans Ouémé où cette activité était plus pratiquée par les femmes $(\mathrm{P}<$ 0,001).

Les éleveurs de porcs étaient sur l'ensemble des départements par ordre d'importance des artisans, des agriculteurs, des ménagères, des retraités, des commerçants, des élèves et étudiants, des salariés et des agroéleveurs, avec une 
différence hautement significative entre départements $(\mathrm{P}<0,001)$. Les artisans étaient majoritaires dans le Plateau et dans le Littoral alors que dans l'Ouémé, les ménagères étaient dominantes $(73 \%)$. Les éleveurs de porcs du Mono et du Zou étaient en majorité des agriculteurs tandis que ceux du Couffo étaient dominés par les élèves et étudiants. La grande majorité des éleveurs (95\%) prenait l'élevage de porcs pour une activité secondaire.

\section{Niveau d'instruction des éleveurs de porcs au Sud du Bénin}

La Figure 3 présente le niveau d'instruction des éleveurs de porcs au SudBénin. La plupart des éleveurs sont scolarisés avec des niveaux d'instruction par ordre d'importance primaire, secondaire et supérieur. Les éleveurs du niveau secondaire étaient majoritaires dans les départements de l'Atlantique et du Littoral tandis que dans les autres départements, ceux du niveau primaire étaient majoritaires. Les éleveurs de porcs du Plateau étaient moins scolarisés.

Niveau de prospérité et situation matrimoniale des éleveurs de porcs au Sud du Bénin

Les éleveurs de porcs (Figure 4) ont présenté trois différents statuts socioéconomiques dont les niveaux de prospérité pauvre, moyen et riche. Le niveau de prospérité pauvre caractérise les éleveurs dont le revenu mensuel (issu de toute activité principale et secondaire) est inférieur à 100000 FCFA. Ceux-ci sont largement représentés dans les départements de Couffo, Ouémé, Plateau, Zou et Mono. Le niveau de prospérité moyen définit les éleveurs dont le revenu mensuel est situé entre 100000 et 200000 FCFA. Les éleveurs de cette catégorie socioéconomique sont majoritaires dans les départements du Littoral (73\%) et de l'Atlantique (56\%). Ceux dont le revenu mensuel est supérieur à 200000 FCFA (niveau de prospérité riche) ont été rencontrés seulement dans les départements du mono, du Zou et du Plateau mais en nombre très réduit. Quant à la situation matrimoniale, les éleveurs de porcs dans tous les départements étaient majoritairement mariés (Figure 5). Toutefois, des veufs/veuves et des célibataires ont été également rencontrés mais en proportions très réduites.

\section{Motivation vis-à-vis du porc, statut des éleveurs et constitution du troupeau}

Les éleveurs du Sud-Bénin étaient motivés pour l'élevage de porcs à cause de leur rusticité, leur prolificité et pour des raisons culturelles (Tableau 2). La rusticité était la prépondérante motivation de l'élevage de porcs dans le Littoral, le Mono et le Couffo, tandis que la prolificité des porcs était déterminante pour les éleveurs du l'Ouémé et $\mathrm{du}$ Zou $(\mathrm{P}<0,001)$. Les animaux étaient une propriété exclusive pour $94 \%$ des éleveurs qui les ont acquis par achat ou héritage contre $6 \%$ en propriété partielle (animaux acquis par le confiage). Les propriétaires des animaux étaient des chefs de ménage, des épouses, des enfants et autres (amis ou alliés). Les chefs de ménage ont dominé dans tous les départements sauf dans l'Ouémé où les épouses propriétaires d'animaux étaient dominantes.

\section{Age, expérience des éleveurs et surface cultivée}

L'âge des éleveurs a varié de 33 à 58 ans $(\mathrm{P}<0,001)$. Les éleveurs du département de l'Atlantique étaient les plus âgés tandis que ceux du département de Couffo étaient les plus jeunes (Tableau 3). Le nombre d'années d'expériences a varié entre 10 et 20 ans. Les élevages de porcs du département du Zou étaient constitués de jeunes exploitations, tandis que ceux du département de l'Ouémé étaient plus anciens (Tableau 3). La plupart des éleveurs associent l'agriculture à l'élevage de porcs. L'emblavure de la production agricole a varié d'un département à un autre $(\mathrm{P}<0,001)$. Cette association de l'agriculture à l'élevage de porcs a été prépondérante par ordre d'importance dans les départements du Zou, de Plateau, de Mono et du Couffo où la surface cultivée varie d'environ de 1 à 3 ha par producteur (Tableau 3). 
Races de porcs élevées au Sud du Bénin et autres espèces animales associées

Les races de porcs élevées étaient des races locales, large-white, landrace et les croisés (Tableau 4). L'élevage des porcs locaux était dominant dans les départements de Mono, de Couffo, de Littoral et de l'Atlantique. Dans l'Ouémé, la race dominante était le large-white alors que les croisés étaient dominants dans le Zou et le Plateau. Certains éleveurs gardent deux à trois races de porcs différentes au sein de leur troupeau afin d'obtenir des produits croisés. Les espèces animales les plus associées à l'élevage de porcs étaient les ruminants et les volailles en particulier les poulets. L'aulacode et le lapin y étaient moins associés $(\mathrm{p}<0,05)$.

Les produits agricoles cultivés par les éleveurs de porcs au Sud du Bénin

Il existe une grande diversité de cultures pratiquées par les porciculteurs d'un département à l'autre (Tableau 5). La culture du maïs et du manioc était la plus associée à la production porcine par les éleveurs. Le palmier à huile était plus cultivé par les éleveurs de porcs du Plateau et du Mono. Quant à l'arachide, elle était cultivée dans le Zou, le Mono et le Couffo. Les produits maraîchers tels que la tomate, le piment et autres (légumes, carotte, chou) n'étaient produits que par les éleveurs des départements du Littoral et de l'Ouémé. La canne à sucre n'était cultivée que par les éleveurs de l'Ouémé. Parmi les éleveurs enquêtés, certains pratiquaient la production de plusieurs produits agricoles à la fois.

\section{Structure et taille des troupeaux de porcs au Sud du Bénin}

L'effectif moyen des troupeaux de porcs a varié de 10 à 23 têtes par exploitation (Tableau 6). Dans les milieux d'étude, l'effectif moyen des truies a varié de 2,80 à 3,87 têtes dont 1 à 2 truies gestantes. Ceux des truies vides et des truies allaitantes ont été en moyenne inférieurs à 2 têtes par troupeau dans toute la zone d'étude. L'effectif moyen des porcelets sevrés a varié d'un département à un autre et a été plus important que celui des porcelets non sevrés. Le nombre moyen de verrats par cheptel a varié entre 0 et 2 têtes. $\mathrm{Vu}$ la grande variabilité des effectifs entre élevage, aucune différence significative n'a été observée entre les effectifs moyens des animaux par classe d'âge et par département.

Variation de la taille du troupeau suivant la profession et le sexe de l'éleveur de porcs au Sud du Bénin

L'effectif des animaux par troupeau a significativement varié en fonction de la profession de l'éleveur $(\mathrm{P}<0,05)$. En effet, les éleveurs qui disposent du plus grand effectif de porcs sont les agents retraités et les salariés suivis des agriculteurs et des artisans. Ceux des autres professions ont présenté des effectifs moins importants (Figure 6). Le Tableau 7 montre que le sexe de l'éleveur a été également source de variation de la taille $\mathrm{du}$ troupeau de porcs au Sud-Bénin $(\mathrm{P}<$ 0,001). En effet, les hommes avaient les effectifs moyens de porcs les plus élevés (19,04 têtes) contre des effectifs relativement faibles chez les femmes (11,86 têtes).

Choix de la race de porcs élevée suivant la profession et le sexe de l'éleveur au Sud du Bénin

Tous les groupes socioprofessionnels pratiquent l'élevage d'au moins trois races de porcs (locale, large-white et croisée) exceptés les agroéleveurs, les élèves et étudiants qui, respectivement n'ont élevé que les croisés et les porcs locaux. La Landrace n'a été élevée que par les artisans et les retraités (Figure 7). Quant à la distribution des races de porcs suivant le sexe de l'éleveur, les hommes ont élevé toutes les races de porcs rencontrées dans le Sud-Bénin avec comme ordre de préférence la race locale, suivie des croisés, ensuite de la large-white et quelquefois la Landrace. La préférence des femmes va vers le porc local, suivi de la Large-white et en dernier choix les croisés. Aucun éleveur de sexe féminin n'a élevé la Landrace (Figure 8). 


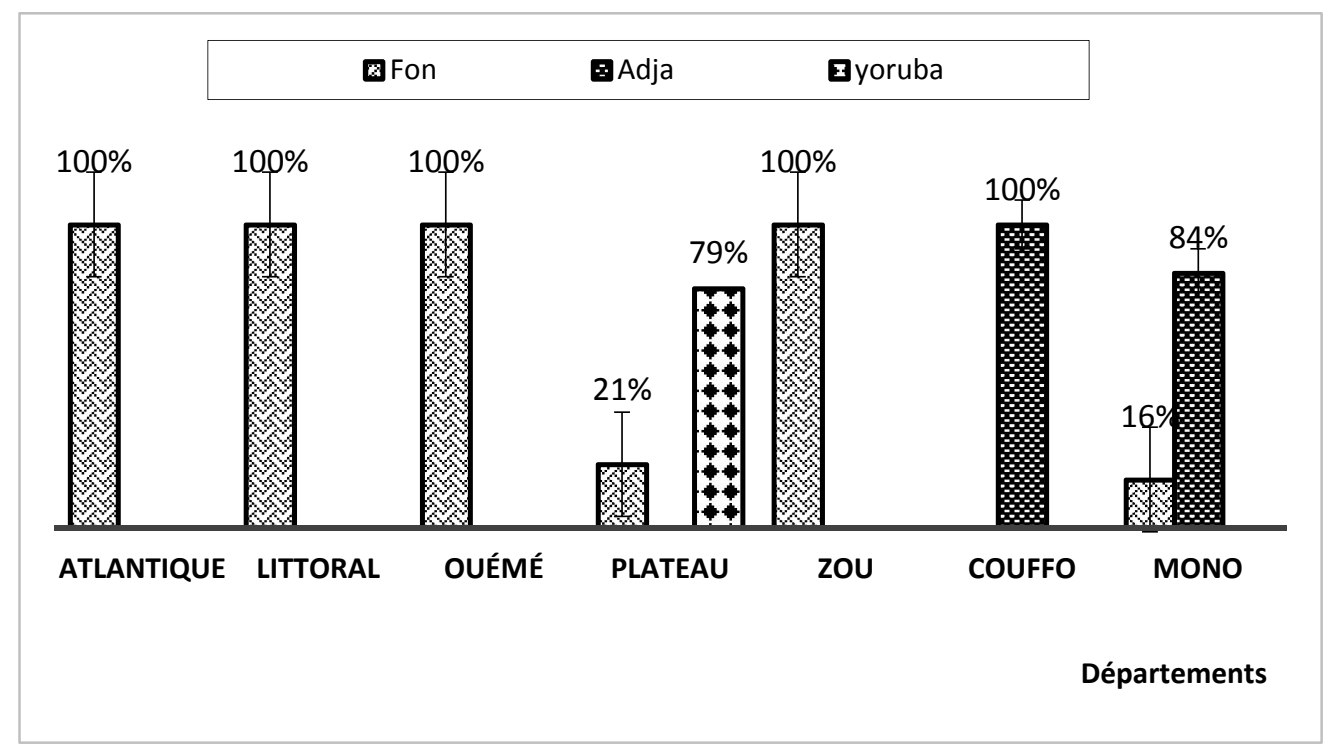

Figure 2 : Groupes sociolinguistiques des éleveurs de porcs au Sud du Bénin.

Tableau 1 : Sexe et groupes socioprofessionnels des éleveurs de porcs au Sud-Bénin.

\begin{tabular}{|c|c|c|c|c|c|c|c|c|c|}
\hline Variables & & Atlantique & Littoral & Ouémé & Plateau & Zou & Couffo & Mono & Test \\
\hline \multirow{2}{*}{ Sexe $(\%)$} & masculin & $93 a$ & $87 \mathrm{a}$ & $33 b$ & $93 \mathrm{a}$ & $93 a$ & $87 \mathrm{a}$ & $67 \mathrm{c}$ & \multirow{2}{*}{$* * *$} \\
\hline & Féminin & $7 a$ & $13 b$ & $67 \mathrm{c}$ & $7 a$ & $7 a$ & $13 b$ & $33 d$ & \\
\hline \multirow{8}{*}{$\begin{array}{c}\text { Activités } \\
\text { principales } \\
(\%)\end{array}$} & Agriculture & $7 \mathrm{a}$ & $27 b$ & $7 \mathrm{a}$ & $13 \mathrm{c}$ & $40 d$ & $13 \mathrm{c}$ & $53 \mathrm{e}$ & \multirow{8}{*}{$* * *$} \\
\hline & Agroélevage & $27 \mathrm{a}$ & 0 & 0 & 0 & $7 b$ & 0 & 0 & \\
\hline & Ménage & 0 & $13 a$ & $73 b$ & $7 \mathrm{c}$ & 0 & $7 \mathrm{c}$ & $13 a$ & \\
\hline & Artisanat & 0 & $47 \mathrm{a}$ & $13 b$ & $60 \mathrm{c}$ & $27 \mathrm{~d}$ & $27 d$ & $13 b$ & \\
\hline & $\begin{array}{l}\text { Petit } \\
\text { commerce }\end{array}$ & $7 \mathrm{a}$ & 0 & $7 \mathrm{a}$ & $7 \mathrm{a}$ & $20 \mathrm{~b}$ & 0 & $13 \mathrm{c}$ & \\
\hline & Salarié & $20 \mathrm{a}$ & $7 b$ & 0 & $13 \mathrm{c}$ & 0 & 0 & 0 & \\
\hline & Retraité & $40 \mathrm{a}$ & $7 b$ & $7 b$ & 0 & $7 b$ & 0 & $7 b$ & \\
\hline & Elève/étudiant & 0 & 0 & 0 & 0 & 0 & 53 & 0 & \\
\hline \multirow{4}{*}{$\begin{array}{c}\text { Activités } \\
\text { secondaires } \\
(\%)\end{array}$} & Agriculture & $13 \mathrm{a}$ & 0 & $13 \mathrm{a}$ & $13 \mathrm{a}$ & $47 b$ & $13 \mathrm{a}$ & $13 a$ & \multirow{4}{*}{$* *$} \\
\hline & Agroélevage & 7 & 7 & 0 & 0 & 0 & 7 & 7 & \\
\hline & Elevage & $93 a$ & $93 a$ & $100 b$ & $100 \mathrm{~b}$ & $93 a$ & $93 a$ & $93 \mathrm{a}$ & \\
\hline & $\begin{array}{l}\text { Petit } \\
\text { commerce }\end{array}$ & 0 & 0 & $40 a$ & $13 b$ & $7 \mathrm{c}$ & 0 & 0 & \\
\hline
\end{tabular}

***: Différence significative au seuil de $0,1 \%, * *$ : Différence significative au seuil de $1 \%$. Les chiffres accompagnés d'une même lettre sur la même ligne ne présentent aucune différence significative. 




Figure 3 : Niveau d'instruction des éleveurs de porcs au Sud du Bénin.

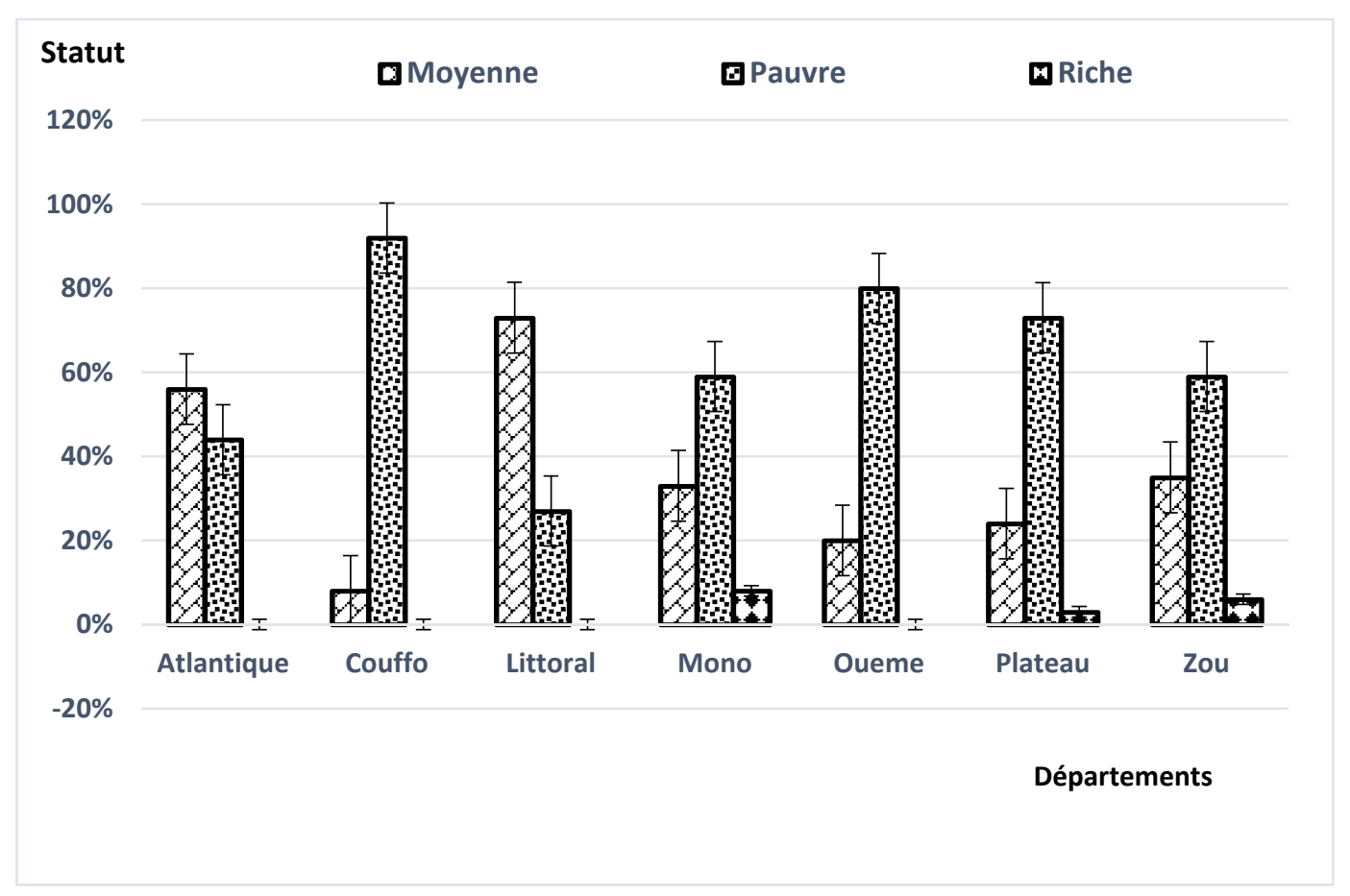

Figure 4 : Statut socioéconomique des éleveurs de porcs au Sud du Bénin. 


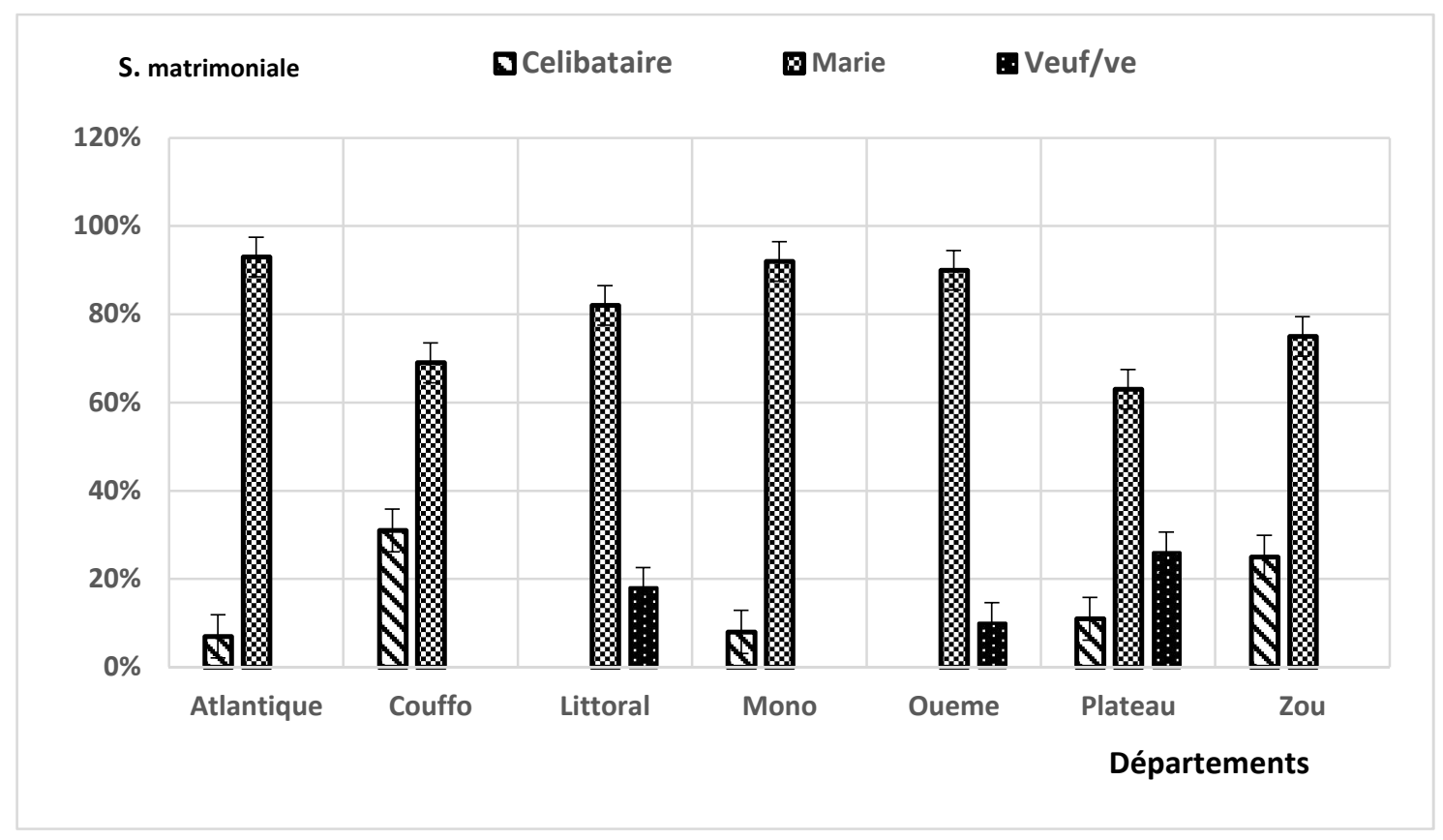

Figure 5 : Situation matrimoniale des éleveurs de porcs au Sud du Bénin.

Tableau 2 : Motivation, statut du propriétaire et constitution du troupeau de porcs.

\begin{tabular}{|c|c|c|c|c|c|c|c|c|c|}
\hline \multicolumn{2}{|c|}{ Variables } & Atlantique & Littoral & Ouémé & Plateau & Zou & Couffo & Mono & Test \\
\hline \multirow{3}{*}{$\begin{array}{c}\text { Motivation } \\
(\%)\end{array}$} & Productivité & $73 a$ & $40 \mathrm{~b}$ & $100 \mathrm{c}$ & $60 d$ & $100 \mathrm{c}$ & $20 \mathrm{e}$ & $7 f$ & \\
\hline & Rusticité & $60 \mathrm{a}$ & $80 \mathrm{~b}$ & $20 \mathrm{c}$ & $47 d$ & $60 \mathrm{a}$ & $87 \mathrm{e}$ & $100 \mathrm{f}$ & $* * *$ \\
\hline & Tradition & 0 & 0 & 0 & 0 & 13 & 0 & 0 & \\
\hline \multirow{2}{*}{$\begin{array}{l}\text { Type de } \\
\text { propriété } \\
(\%)\end{array}$} & Exclusive & $100 \mathrm{a}$ & $87 \mathrm{~b}$ & $100 \mathrm{a}$ & $100 \mathrm{a}$ & $100 \mathrm{a}$ & $80 c$ & $93 a$ & $*$ \\
\hline & Partielle & 0 & 13 & 0 & 0 & 0 & 20 & 7 & \\
\hline \multirow{4}{*}{$\begin{array}{c}\text { Propriétaire } \\
(\%)\end{array}$} & $\begin{array}{l}\text { Chef } \\
\text { ménage }\end{array}$ & $93 a$ & $80 \mathrm{~b}$ & $33 c$ & $93 a$ & $80 \mathrm{~b}$ & $40 d$ & $60 \mathrm{e}$ & \\
\hline & Epouse & $7 \mathrm{a}$ & $13 b$ & $67 \mathrm{c}$ & $7 \mathrm{a}$ & $13 b$ & $13 b$ & $33 d$ & $* * *$ \\
\hline & Enfant & 0 & $7 \mathrm{a}$ & 0 & 0 & $7 \mathrm{a}$ & $33 b$ & 0 & \\
\hline & Autres & 0 & 0 & 0 & 0 & 0 & 13 & 7 & \\
\hline \multirow{3}{*}{$\begin{array}{l}\text { Constitution } \\
\text { du troupeau } \\
(\%)\end{array}$} & Achat & $100 \mathrm{a}$ & $93 a$ & $100 \mathrm{a}$ & $100 \mathrm{a}$ & $93 a$ & $80 \mathrm{~b}$ & $93 a$ & \\
\hline & Confiage & 0 & $7 \mathrm{a}$ & 0 & 0 & 0 & $20 \mathrm{~b}$ & $7 \mathrm{a}$ & NS \\
\hline & Don & 0 & 0 & 0 & 0 & 7 & 0 & 0 & \\
\hline
\end{tabular}


Tableau 3 : Age et expérience des éleveurs de porcs au Sud du Bénin et surface de production agricole emblavée.

\begin{tabular}{lcccccccc}
\hline Variables & Atlantique & Littoral & Ouémé & Plateau & Zou & Couffo & Mono & Test \\
\hline Age de l'éleveur (ans) & $58 \mathrm{a}$ & $46 \mathrm{~b}$ & $51 \mathrm{c}$ & $41 \mathrm{~d}$ & $44 \mathrm{~b}$ & $33 \mathrm{e}$ & $45 \mathrm{~b}$ & $* * *$ \\
\hline $\begin{array}{l}\text { Expérience des } \\
\text { éleveurs (ans) }\end{array}$ & $16 \mathrm{a}$ & $19 \mathrm{~b}$ & $20 \mathrm{~b}$ & $15 \mathrm{a}$ & $10 \mathrm{c}$ & $18 \mathrm{~b}$ & $16 \mathrm{a}$ & $\mathrm{NS}$ \\
\hline Surface cultivée (ha) & $0,47 \mathrm{a}$ & $0,13 \mathrm{~b}$ & $0,2 \mathrm{c}$ & $1,73 \mathrm{~d}$ & $2,73 \mathrm{e}$ & $0,93 \mathrm{f}$ & $1,07 \mathrm{~g}$ & $* * *$ \\
\hline
\end{tabular}

***: Différence significative au seuil de $0,1 \%$. Les chiffres affectés d'une même lettre sur la même ligne ne présentent aucune différence significative.

Tableau 4 : Races de porcs élevées au Sud du Bénin et autres espèces associées.

\begin{tabular}{|c|c|c|c|c|c|c|c|c|c|}
\hline Variabl & & Atlantique & Littoral & Ouémé & Plateau & Zou & Couffo & Mono & Test \\
\hline \multirow{4}{*}{$\begin{array}{c}\text { Races de porcs } \\
\text { élevées }(\%)\end{array}$} & Locale & $60 \mathrm{a}$ & $73 b$ & $20 \mathrm{c}$ & $47 d$ & $20 c$ & $87 \mathrm{e}$ & $100 \mathrm{f}$ & \multirow{4}{*}{$* * *$} \\
\hline & $\begin{array}{l}\text { Large- } \\
\text { white }\end{array}$ & $20 \mathrm{a}$ & $20 \mathrm{a}$ & $93 b$ & $13 \mathrm{c}$ & $20 \mathrm{a}$ & $20 \mathrm{a}$ & $7 d$ & \\
\hline & Landrace & 7 & 0 & 0 & 0 & 0 & 7 & 0 & \\
\hline & Croisée & $73 a$ & $27 b$ & $7 \mathrm{c}$ & $53 d$ & $87 \mathrm{e}$ & $7 \mathrm{c}$ & $7 \mathrm{c}$ & \\
\hline \multirow{8}{*}{$\begin{array}{l}\text { Autres espèces } \\
\text { associées }(\%)\end{array}$} & Ruminant & $40 a$ & $20 \mathrm{~b}$ & $20 \mathrm{~b}$ & $60 \mathrm{c}$ & $67 d$ & $40 \mathrm{a}$ & $27 \mathrm{e}$ & \multirow{8}{*}{$* *$} \\
\hline & Poulet & $67 a$ & $27 b$ & $7 \mathrm{c}$ & $13 d$ & $67 a$ & $27 \mathrm{~b}$ & $47 \mathrm{e}$ & \\
\hline & Canard & $27 \mathrm{a}$ & 0 & $7 b$ & 0 & $13 \mathrm{c}$ & $7 b$ & 0 & \\
\hline & Pintade & $13 \mathrm{a}$ & 0 & 0 & 0 & $13 \mathrm{a}$ & $7 b$ & 0 & \\
\hline & Dindon & $7 \mathrm{a}$ & 0 & 0 & 0 & $33 b$ & $7 \mathrm{a}$ & 0 & \\
\hline & Lapin & 7 & 0 & 0 & 0 & 0 & 0 & 0 & \\
\hline & Aulacode & 0 & 0 & 0 & 7 & 7 & 0 & 0 & \\
\hline & Aucune & $20 \mathrm{a}$ & $53 b$ & $80 \mathrm{c}$ & $33 d$ & $20 \mathrm{a}$ & $47 \mathrm{e}$ & $33 d$ & \\
\hline
\end{tabular}

***: Différence significative au seuil de $0,1 \%, * *$ : Différence significative au seuil de $1 \%$. Les chiffres accompagnés d'une même lettre sur la même ligne ne présentent aucune différence significative.

Tableau 5 : Produits agricoles cultivés par les éleveurs de porcs au Sud du Bénin.

\begin{tabular}{lcccccccc}
\hline \multicolumn{1}{c}{ Variables } & Atlantique & Littoral & Ouémé & Plateau & Zou & Couffo & Mono & Test \\
\hline Maïs (\%) & $33 \mathrm{a}$ & 0 & $7 \mathrm{~b}$ & $47 \mathrm{c}$ & $93 \mathrm{~d}$ & $40 \mathrm{e}$ & $67 \mathrm{f}$ & \\
Manioc (\%) & $27 \mathrm{a}$ & 0 & $13 \mathrm{~b}$ & $47 \mathrm{c}$ & $100 \mathrm{~d}$ & $33 \mathrm{e}$ & $67 \mathrm{f}$ & \\
Haricot (\%) & $7 \mathrm{a}$ & 0 & 0 & $20 \mathrm{~b}$ & $20 \mathrm{~b}$ & $27 \mathrm{c}$ & $60 \mathrm{~d}$ & \\
Arachide (\%) & 0 & 0 & 0 & 0 & $40 \mathrm{a}$ & $27 \mathrm{~b}$ & $33 \mathrm{c}$ & \\
Palmier à huile (\%) & $13 \mathrm{a}$ & 0 & $7 \mathrm{~b}$ & $27 \mathrm{c}$ & 0 & 0 & $27 \mathrm{c}$ & $* * *$ \\
Tomate (\%) & 0 & $33 \mathrm{a}$ & $20 \mathrm{~b}$ & 0 & $7 \mathrm{c}$ & 0 & 0 & \\
Piment (\%) & 0 & $33 \mathrm{a}$ & $7 \mathrm{~b}$ & 0 & 0 & 0 & 0 & \\
Canne à sucre (\%) & 0 & 0 & 13 & 0 & 0 & 0 & 0 & \\
Autres produits & & & & & & & & \\
maraîchers (\%) & 0 & $33 \mathrm{a}$ & $7 \mathrm{~b}$ & 0 & $7 \mathrm{~b}$ & 0 & 0 & \\
Aucun (\%) & $60 \mathrm{a}$ & $67 \mathrm{~b}$ & $80 \mathrm{c}$ & $47 \mathrm{~d}$ & 0 & $60 \mathrm{a}$ & $27 \mathrm{e}$ & \\
\hline
\end{tabular}

***: Différence significative au seuil de $0,1 \%$. Les chiffres accompagnés d'une même lettre sur la même ligne ne présentent aucune différence significative. 
Tableau 6 : Structure et taille du troupeau de porcs au Sud du Bénin.

\begin{tabular}{|c|c|c|c|c|c|c|c|c|c|}
\hline \multicolumn{2}{|c|}{ Variables } & Atlantique & Littoral & Ouémé & Plateau & Zou & Couffo & Mono & Test \\
\hline \multirow{8}{*}{$\begin{array}{l}\text { Structure } \\
\text { et taille du } \\
\text { troupeau } \\
\text { (Têtes) }\end{array}$} & $\begin{array}{l}\text { Effectif de } \\
\text { truies }\end{array}$ & $3,6 a$ & $3,87 \mathrm{a}$ & $3,67 \mathrm{a}$ & $2 b$ & $2,8 \mathrm{c}$ & $3,07 \mathrm{a}$ & $2,73 c$ & NS \\
\hline & Truies vides & $1,4 a$ & $0,87 b$ & $1,27 \mathrm{a}$ & $0,4 \mathrm{c}$ & $1,2 \mathrm{a}$ & $1,27 \mathrm{a}$ & $1,13 \mathrm{a}$ & NS \\
\hline & $\begin{array}{l}\text { Truies } \\
\text { gestantes }\end{array}$ & $1,47 \mathrm{a}$ & $1,93 a$ & $2,07 \mathrm{a}$ & $1,13 b$ & $1,07 b$ & $1,2 b$ & $1,07 b$ & NS \\
\hline & $\begin{array}{l}\text { Truies } \\
\text { allaitantes }\end{array}$ & $0,87 \mathrm{a}$ & $1,07 \mathrm{a}$ & $0,27 b$ & $0,47 \mathrm{c}$ & $0,53 \mathrm{c}$ & $0,6 \mathrm{c}$ & $0,6 \mathrm{c}$ & NS \\
\hline & Porcs sevrés & $12,87 \mathrm{a}$ & $12,47 \mathrm{a}$ & $9,8 b$ & $5,53 \mathrm{c}$ & $8,93 b$ & $7,27 \mathrm{~d}$ & $11,6 a$ & NS \\
\hline & $\begin{array}{l}\text { Porcs non } \\
\text { sevrés }\end{array}$ & $4,53 \mathrm{a}$ & $5,87 \mathrm{a}$ & $2 b$ & $1,87 \mathrm{~b}$ & $4,93 a$ & $2,73 c$ & $3,33 c$ & NS \\
\hline & Verrats & $1 \mathrm{a}$ & $0,93 \mathrm{a}$ & $0,73 \mathrm{a}$ & $0,8 \mathrm{a}$ & $0,93 a$ & $1,33 b$ & $1,6 b$ & NS \\
\hline & Effectif total & $22,07 \mathrm{a}$ & $23,07 \mathrm{a}$ & $16,2 b$ & $10,13 c$ & $17,53 \mathrm{~b}$ & $14,4 d$ & $19,33 b$ & $* *$ \\
\hline
\end{tabular}

NS : Différence non significative au seuil de 5\%. Les chiffres accompagnés d'une même lettre sur la même ligne ne présentent aucune différence significative.



Figure 6 : Variation de la taille du troupeau suivant la profession de l'éleveur au Sud du Bénin.

Tableau 7 : Variation de la taille du troupeau suivant le sexe de l'éleveur de porcs au Sud du Bénin.

\begin{tabular}{lcccc} 
& Effectif par sexe & Taille du troupeau & Ecart-type & $\mathbf{p}>\mathbf{F}$ \\
\hline Masculin & 83 & 19,04 & 18,62 & \\
Féminin & 22 & 11,86 & 12,83 & $\mathrm{P}<0,001$ \\
Total & 105 & 17,53 & 17,76 & \\
\hline
\end{tabular}




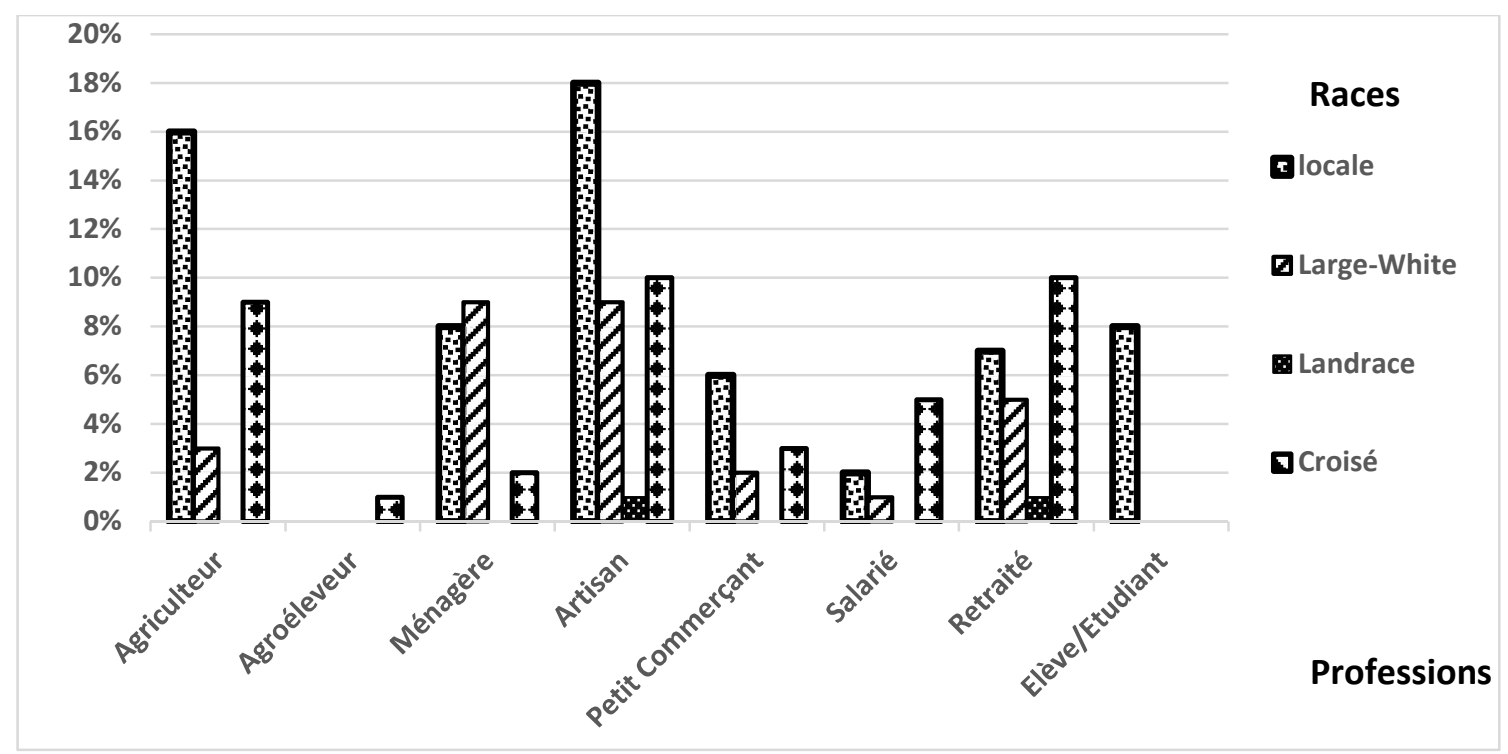

Figure 7 : Distribution des races de porcs selon le groupe socioprofessionnel au Sud du Bénin.



Figure 8 : Distribution des races de porcs suivant le sexe de l'éleveur au Sud du Bénin. 
DISCUSSION

Groupe sociolinguistique des éleveurs de pores

Cette étude, montre que l'élevage de porc est pratiqué par plusieurs groupes sociolinguistiques du Sud-Bénin. Ces résultats sont conformes à ceux de Mopaté et KaboréZoungrana (2009) à N'Djaména où plusieurs ethnies pratiquent l'élevage de porcs. Les trois grands groupes sociolinguistiques auxquels appartiennent les éleveurs du Sud-Bénin sont les Fon, les Adja et les Yoruba. Cette variation des groupes sociolinguistiques indique que l'élevage de porcs n'est pas l'apanage d'un seul groupe sociolinguistique. Cependant, les Fon sont largement dominants certainement du fait qu'ils sont omniprésents dans tous les départements sauf le Couffo où les éleveurs appartiennent presqu'exclusivement au groupe sociolinguistique Adja originaires de cette aire géographique. En plus de l'omniprésence des Fon dans cinq des sept départements d'élevage de porcs, une autre explication de la dominance des Fon dans l'élevage porcin peut être l'importance de l'espèce porcine dans leur culture et dans leur habitude alimentaire.

\section{Le sexe des éleveurs de porcs}

L'élevage de porcs est une activité principalement pratiquée par les hommes dans le sud Bénin (79\%). Ces résultats sont conformes à ceux obtenus par Mopaté (2008) à N'Djaména (73\%), Ndébi et al. (2009) au Cameroun (85\%) et Houndonougbo et al. (2012) à Adjarra au Sud-Est du Bénin (97\%). Le faible taux de participation des femmes à la production porcine est lié d'une part au fait que le porc, compte tenu de son agressivité est un animal difficile à manipuler et d'autre part au caractère sacré de l'espèce porcine dans les traditions au Sud du Bénin. Mais quoique faiblement représentées, l'implication des femmes dans la production porcine au Sud du Bénin révèle la nécessité de la prise en compte de l'approche genre dans la politique de promotion de la filière porcine au Bénin.
Le groupe socioprofessionnel des éleveurs de porcs

Les éleveurs de porc sont pour la plupart des agriculteurs, des éleveurs, des commerçants, des ménagères, des artisans, des salariés et autres (élèves et étudiants). Cela montre que l'élevage du porc est une activité secondaire des producteurs et se fait dans le but de diversifier les sources de revenus en vue de sécuriser les familles. Ces résultats sont conformes à ceux obtenus par Mopaté (2002) à N'Djaména au Tchad et ceux observés par Youssao et al. (2008) au Bénin relatant que l'élevage du porc est une activité économique complémentaire de presque toutes les catégories socioprofessionnelles. La diversité de groupes socioprofessionnels pratiquant l'élevage porcin montre que cette activité est une alternative qui s'impose aux ménages lorsque les conditions économiques deviennent difficiles (Spore, 2000 ; Ali et al., 2003 ; Abdou et al., 2004 ; Dongmo et al., 2005). Sur le plan macroéconomique, cette diversité des groupes socioprofessionnels et socioculturels des éleveurs de porcs montre que l'investissement du pouvoir politique dans ce sous-secteur aura à coup sûr un impact positif dans la lutte contre la pauvreté et l'insécurité alimentaire au Bénin. L'association de l'agriculture à l'élevage de porcs révèle l'importance de l'interaction entre les deux sous-secteurs.

\section{Niveau d'instruction et statut socioéconomique des éleveurs de porcs}

Les résultats de la présente étude ont montré que l'élevage de porcs au Sud du Bénin est une activité génératrice de revenus de grande importance dans la vie des personnes non instruites (analphabètes) et à faible revenus (niveau de prospérité pauvre) qui représentent la majorité des éleveurs. Ainsi, le niveau d'instruction et le statut socioéconomique ne constituent guère une barrière à la pratique de l'élevage de porcs au Sud du Bénin. La production porcine constitue pour les populations rurale, urbaine et périurbaine un moyen de subsistance et de lutte contre la pauvreté (FAO, 2012). Cette 
grande envergure que connaît l'élevage de porcs au Sud du Bénin est due au fait que dans cette région du pays, les interdits religieux notamment ceux de l'Islam ont très peu d'influence à cause de la minorité des musulmans. Le caractère moins exigeant de cet élevage ainsi que sa rentabilité le rendent également accessible à toutes les couches socioéconomiques (Kamuanga et al., 2008).

\section{Taille et structure des troupeaux de porcs}

Les effectifs moyens des troupeaux de porcs obtenus dans le Littoral (23,07 têtes) et dans l'Atlantique (22,07 têtes) au cours de cette étude sont au-dessus de ceux observés par Mopaté et Kaboré-Zoungrana (2010) à N'Djaména respectivement dans le $1^{\text {er }}$ arrondissement $(15,4$ têtes $)$, le $3^{\text {ème }}$ arrondissement (18,5 têtes), le $6^{\text {ème }}$ arrondissement $(12,1$ têtes $)$, le $7^{\text {ème }}$ arrondissement $(14,9$ têtes $)$ et le $9^{\text {ème }}$ arrondissement (12,09 têtes). Il en est de même pour les résultats obtenus par Youssao et al. (2008) en zone périurbaine de Cotonou et d'Abomey-Calavi (19 têtes) et ceux de Houndonougbo et al. (2012) dans la commune d'Adjarra au Sud-Est du Bénin (11,4 têtes). Cette différence s'explique par le fait que Cotonou (Littoral) et Abomey-Calavi (Atlantique) sont des villes cosmopolites qui regorgent assez de consommateurs de la viande de porcs. Cela peut aussi s'expliquer par la disponibilité de ressources comme les sous-produits provenant des grandes industries agroalimentaires et des sociétés de distribution des matières premières et d'intrants de production animale installées dans ces deux communes. Ainsi, les faibles effectifs moyens enregistrés dans les autres départements seraient liés au statut socioéconomique ou aux difficultés des éleveurs à accéder aux intrants et aux matières premières utilisés dans l'élevage des animaux domestiques. Bien que Sémè Kpodji (Ouémé) soit une commune voisine de Cotonou, la taille moyenne des troupeaux de porcs est restée faible. Ces résultats confirment l'influence du sexe de l'éleveur sur la taille du troupeau (Tableau 8) étant donné que les éleveurs de cette commune sont dominés par les femmes (73\%). Les effectifs moyens de truies qui varient de 2 à 3,87 têtes par troupeau, sont voisins de ceux obtenus par Houndonougbo et al. (2012) à Adjarra au Bénin (3,1 truies), par Mopaté (2008) à N'Djaména (2,76 truies), par Mopaté et Kaboré-Zoungrana (2010) à Bangui (3,9 truies), et par Youssao et al. (2008) dans les zones périurbaines de Cotonou et d'AbomeyCalavi au Bénin (4,18 truies). En outre, les effectifs de truies de la présente étude sont largement supérieurs à ceux obtenus par Nonfon et al. (1994) au Sud du Bénin où l'effectif moyen le plus élevé de truies par troupeau était 0,71 truies. Cette différence peut s'expliquer par l'augmentation constante de la demande en viande de porcs qui entre de plus en plus dans l'habitude alimentaire de la population. L'absence de verrats dans certains troupeaux de porcs au cours de la présente étude confirme les résultats de Halimani et al. (2012) en Afrique du Sud et ceux de Houndonougbo et al. (2012), Youssao et al. (2008) et de Nonfon et al. (1994) au Bénin selon lesquels les éleveurs ne disposant pas de verrats font l'emprunt ou en achètent pour la circonstance et procèdent ensuite à la vente après les avoir castrés et engraissés. Ce manque de verrat dans certains élevages est un facteur susceptible de conduire à un degré élevé de consanguinité au sein de la population porcine au sud du Bénin. Ces difficultés des éleveurs participent également à la persistance du mode d'élevage en divagation ou mixte observée dans la plupart des zones d'étude où le brassage entre troupeaux en liberté offre plus de chances aux femelles en chaleur de se faire saillir par des verrats d'autres cheptels.

\section{Variation de la taille du troupeau suivant le groupe socioprofessionnel et le sexe de l'éleveur}

L'effectif moyen du troupeau est significativement influencé par la profession de l'éleveur $(\mathrm{P}<0,05)$. Ainsi, les retraités et les salariés détiennent les effectifs les plus importants de porcs, respectivement 32,8 têtes 
et 23,7 têtes tandis que les effectifs les plus faibles sont détenus par les élèves et étudiants $(8,13$ têtes) ainsi que les petits commerçants (9,25 têtes). Cela peut s'expliquer par le fait que les retraités sont plus disponibles à s'occuper des animaux à plein temps. De plus, leurs revenus mensuels quelque peu réduits par l'admission à la retraite, ces derniers n'ont eu pour solution que d'investir dans l'élevage de porcs comme moyen de diversification et d'augmentation de leurs revenus. Les salariés, quant à eux, disposent d'assez de ressources financières pour l'installation des infrastructures d'élevage adéquates et la prise en charge de la gestion technicoéconomique du troupeau. Par contre, les élèves/étudiants et les petits commerçants n'ayant pas assez de moyens sont obligés d'engager un effectif à la taille de leur moyen. Le faible effectif des troupeaux détenus par les femmes est en accord avec les résultats de Herrero et al. (2014). Ces auteurs rapportent que la faible mobilité (possibilité réduite de divagation en quête de d'aliment) des troupeaux de femmes à cause de l'occupation de celles-ci par les travaux ménagers constitue la contrainte majeure qui limite la possession de troupeaux plus larges. Par contre, les résultats de la présente étude sont en désaccord avec ceux de Halimani et al. (2012) en Afrique du Sud où les éleveurs de porcs sont largement dominés par les femmes $(69,7 \%)$ qui détiennent la plus grande part de l'effectif du cheptel porcin.

\section{Choix des races de porcs}

La présente étude a révélé que l'élevage de porcs locaux représente en moyenne $58 \%$ du cheptel porcin au Sud du Bénin comme c'est le cas en Afrique du Sud (Halimani et al., 2012). Les porcs locaux en 1994 représentaient $93,5 \%$ du cheptel porcin au Sud du Bénin (Nonfon et al., 1994). En comparaison aux résultats de la présente étude, on peut estimer la réduction de l'élevage de porc local de $35,5 \%$ de 1994 à 2015 au profit de l'élevage des croisés et des races exotiques. Cette réduction de la proportion des élevages de porc local révèle une sous-utilisation de la race locale, pouvant se traduire par une réduction de leurs effectifs au profit de ceux des races exotiques et des croisés. En effet, la substitution et les croisements entre races locales et exotiques deviennent de plus en plus courants chez les éleveurs sans distinction de sexe, de profession et de groupe sociolinguistique en quête de produits performants. Cette pratique expose le porc local à des risques d'érosion génétique d'où l'importance des mesures de préservation de ces ressources génétiques autochtones au Sud du Bénin.

\section{Conclusion}

Les caractéristiques sociodémographiques des éleveurs de porcs et la structure du cheptel porcin ont été étudiées au Sud du Bénin. Il ressort de cette étude que les éleveurs appartiennent à une grande diversité de groupes sociolinguistiques et socioprofessionnels. Toutes les couches socioéconomiques s'intéressent à l'élevage de porcs sans distinction de sexe et de niveau d'instruction. Ces différentes caractéristiques que présentent les éleveurs de porcs influent sur la structure et la taille du troupeau ainsi que la distribution des races de porcs élevées dans la zone de l'étude. Ainsi, le développement de la production porcine au Bénin aura à coup sûr un impact positif sur la réduction de la pauvreté. A l'issue de la présente étude, il est souhaitable que les investigations soient poursuivies dans le but d'appréhender les modalités de conduite des cheptels et les contraintes majeures qui entravent le développement du sous-secteur élevage de porcs afin d'y opposer des mesures palliatives.

\section{CONFLITS D'INTERETS}

Les auteurs déclarent qu'il n'existe aucun conflit d'intérêts au sujet du présent article.

\section{CONTRIBUTION DES AUTEURS}

Tous les auteurs ont participé à la collecte, au traitement des données et à la rédaction de cet article. 


\section{REFERENCES}

Aboki SS. 2011. Caractéristiques des secteurs de transformation et de consommation de la filière viande porcine dans les Départements de l'Ouémé et du Zou au Bénin. Rapport de fin de formation en Production et Santé Animales. EPAC/UAC. 45 p.

Abdou F, Diao MB, Bastianelli D, Nianogo AJ. 2004. La gestion concertée et durable des filières animales urbaines. In Développement Durable de l'Agriculture Urbaine en Afrique Francophone : Enjeux, Concepts et Méthodes, Smith OB, Moustier P, Mougeot LJA, Abdou F (éds). Cirad-Crdi ; 115-142.

Ali L, Van-Den-Bossche P, Thys E. 2003. Enjeux et contraintes de l'élevage urbain et périurbain des petits ruminants à Maradi au Niger : quel avenir? Revue Elev. Méd. vét. Pays trop., 56(1-2) : 7382.

Atodjinou FTR, Dotcho CDG. 2006. Caractéristiques de l'élevage des porcs locaux dans les élevages périurbains de Cotonou et d'Abomey-Calavi. Diplôme d'Études Agricoles Tropicales, Lycée Mèdji de Sékou, Bénin. 80p.

CuntryStats. 2013. Répartition des animaux vivants selon l'année. Niveau administratif 2, Produits (Têtes). Source: CARDER et Direction de l'élevage, Bénin. www.countrystats.com

Boutonnet J-P, Griffon M, Viallet D. 2000. Compétitivité des productions animales en Afrique subsaharienne et à Madasgascar. Synthèse générale, Direction Générale de la Coopération Internationale, Ministère des Affaires Étrangères, France, Paris. 104p.

Dongmo T, Gockwski J, Hernandez S, Awono LDK, Mbang A, Moudon R. 2005. L'agriculture périurbaine à Yaoundé : ses rapports avec la réduction de la pauvreté, le développement économique, la conservation de la biodiversité et de l'environnement. Tropicultura, 23(3) : 130-135.
FAO. 2012. Pertes et Gaspillage Alimentaire dans le Monde : Ampleur, Causes et Prévention. Etude Menée pour le Congrès International SAVE FOOD. FAO : Rome ; $41 \mathrm{p}$.

Halimani TE, Muchadeyi FC, Chimonyo M, Dzama K. 2012. Some insights into the phenotypic and genetic diversity of indigenous pigs in southern Africa. $S$. Afr. J. Anim. Sci., 42: 4.

Herrero M, Havlik P, McIntire J, Palazzo A, Valin H. 2014. L'avenir de l'élevage africain: Réaliser le potentiel de l'élevage pour la sécurité alimentaire, la réduction de la pauvreté et la protection de l'environnement en Afrique subsaharienne. Bureau du représentant spécial des Nations Unies pour la sécurité alimentaire et nutritionnelle et $\mathrm{du}$ Coordonnateur du système des Nations Unies contre la grippe (UNSIC), Genève, Suisse, $118 \mathrm{p}$.

Houndonougbo MF, Adjolohoun S, Aboh BA, Singbo A, Chrysostome CAAM. 2012. Caractéristiques du système d'élevage porcin au Sud-Est du Bénin. Bulletin de la Recherche Agronomique du Bénin (BRAB), (Numéro spécial Elevage \& Faune) : 15-21.

Kamuanga JBM, Somda J, Sanon Y, Kagoné H. 2008. Élevage et Marché Régional au Sahel et en Afrique de l'Ouest, Potentialités et Défis. (C) CSAO-OCDE / CEDEAO, 2008. Ed. : Club du Sahel et de l'Afrique de l'Ouest/OCDE. 182p.

Mopaté LY. 2002. Productivité des élevages porcins dans les départements du Logone occidental, du Mayo-Dallah et de la Kabia en zone soudanienne du Tchad. Rapport de mission. N'Djamena, Tchad, Lrvz. 15p.

Mopaté LY, Koussou MO. 2002. L'élevage porcin, un élevage ignoré mais pourtant bien implanté dans les agrosystèmes ruraux et périurbains du Tchad. In : Actes du colloque « Savanes africaines : des espaces en mutations, des acteurs face à des nouveaux défis ", Garoua, 
Cameroun, 27-31 mai 2002, Jamin JY, Seyni Boukar L, Floret C, (éds), 9p.

Mopaté LY. 2008. Dynamique des élevages porcins et amélioration de la production en zones urbaine et périurbaine de N'Djaména (Tchad). Doctorat Unique, Université Polytechnique de BoboDioulasso (UPB), Burkina Faso, 246 p.

Mopaté LY, Kaboré-Zoungrana C-Y. 2009. Dynamique des élevages et caractéristiques des producteurs de porcs de la ville de N'Djaména, Tchad. Manuscrit auteur, publié dans "Savanes africaines en développement : innover pour durer, Garoua : Cameroun (2009)", cirad-00472076, version 1 - 9 Apr 2010. $10 \mathrm{p}$.

https://www.researchgate.net/publication /43076211

Mopaté LY, Kaboré-Zoungrana C-Y. 2010. Dynamique des élevages et caractéristiques des producteurs de porcs de la ville de N'Djaména, Tchad. In : Actes du colloque «Savanes africaines en développement : innover pour durer », Jamin, JY, Seyni Boukar L, Floret C, (éds); 20-23 Avril 2009, Garoua, Cameroun, 9p.

Mopaté LY, Koussou MO, Nguertoum ET, Ngo-Tama AC, Lakouetene T, Awa DN, Mal HE. 2010. Caractéristiques et performances des élevages porcins urbains et périurbains des savanes d'Afrique centrale : cas des villes de Garoua, Pala et Bangui. In : Actes du colloque « Savanes africaines en développement : innover pour durer »,
Jamin JY, Seyni-Boukar L, Floret C, (éds); 20-23 Avril 2009, Garoua, Cameroun, 9p.

Nonfon WR, Deka E, Adégbidi A, Codjo B. 1994. Elevage de porc local au Bénin. Rapport I : enquêtes diagnostics sur les systèmes d'élevage et filière de commercialisation. Tome I : système d'élevage. FSA/UNB. 146p.

Ndébi G, Kamajou J, Ongla J. 2009. Analyse des contraintes au développement de la production porcine au Cameroun. Tropicultura, 27(2) 70-76.

Porphyre V. 2009. Enjeux et contraintes des filières porcines en Afrique de l'Ouest. In Grain de sel $n^{\circ}$ 46-47, mars - août 2009. Le dossier des systèmes de production, des enjeux, des défis. $2 \mathrm{p}$.

Youssao AKI, Koutinhouin GB, Kpodékon TM, Yacoubou A, Bonou AG, Adjapka A, Ahounou S, Taiwo R. 2009. Amélioration génétique des performances zootechniques du porc local du Bénin par croisement avec le Large White. Int. J. Biol. Chem. Sci., 3: 653-662.

Spore. 2007. Porc, des atouts sous la menace. Magasine bimestriel du Centre technique de coopération agricole et rurale (CTA), $\mathrm{n}^{\circ} 132 \mathrm{du}$ mois de décembre, $11 \mathrm{p}$.

Youssao AKI, Koutinhouin GB, Kpodékon TM, Bonou AG, Adjakpa A, Dotcho CDG, Atodjinou FTR. 2008. Production porcine et ressources génétiques locales en zone périurbaine de Cotonou et d'Abomey-Calavi au Bénin. Revue Elev. Méd. Vét. Pays Trop., 61(3-4) : 235-243. 